\title{
Clinical Safety and Efficacy of Secondary Prophylactic Pegylated G-CSF in Advanced Pancreatic Cancer Patients Treated with mFOLFIRINOX: A Single-center Retrospective Study
}

Kentaro Yamao ${ }^{1}$, Mamoru Takenaka ${ }^{1}$, Tomoe Yoshikawa ${ }^{1}$, Rei Ishikawa ${ }^{1}$, Ayana Okamoto ${ }^{1}$, Tomohiro Yamazaki ${ }^{1}$, Atsushi Nakai ${ }^{1}$, Shunsuke Omoto ${ }^{1}$, Ken Kamata ${ }^{1}$, Kosuke Minaga ${ }^{1}$, Satoru Hagiwara ${ }^{1}$, Toshiharu Sakurai ${ }^{1}$, Naoshi Nishida ${ }^{1}$, Yasutaka Chiba ${ }^{2}$,

Tomohiro Watanabe ${ }^{1}$ and Masatoshi Kudo ${ }^{1}$

\begin{abstract}
:
Objective Although modified FOLFIRINOX (mFOLFIRINOX, mFFX) is widely used for patients with advanced pancreatic ductal adenocarcinoma (PDAC), maintenance of the standard dose intensity is often difficult due to the high incidence of neutropenic events. Pegylated granulocyte colony-stimulating factor (GCSF) (Peg G) is a long-lasting G-CSF agent that is applicable for prophylaxis against neutropenic complications. The aim of this study was to assess the clinical safety and efficacy of mFFX combined with secondary prophylaxis using Peg $\mathrm{G}$ in advanced PDAC patients.

Methods Advanced PDAC patients who had received more than two cycles of mFFX were analyzed. The clinical safety and efficacy were compared between patients in the Peg G group and those in the non-Peg G group in a retrospective manner.

Results Among 45 patients treated with mFFX, 28 exhibited grade 3-4 neutropenia or febrile neutropenia. Among these 28 patients, 4 who received only 1 or $2 \mathrm{mFFX}$ cycles were excluded from this study. Finally, 11 patients in the Peg G group and 13 in the non-Peg G group were enrolled. The combination therapy with Peg G and mFFX markedly prolonged the progression-free survival compared with the non-Peg G group, and its effects were associated with a reduced incidence of neutropenic events as well as lower rates of dosage reduction, delayed chemotherapy due to neutropenic events and altered blood cell counts after chemotherapy.

Conclusion The scheduled administration of secondary prophylactic Peg G prolonged the progression-free survival in patients treated with mFFX. The combination therapy of Peg G and mFFX may be recommended in patients who exhibit grade 3-4 neutropenic events after prior mFFX cycles.
\end{abstract}

Key words: pegylated G-CSF, mFOLFIRINOX, pancreatic cancer

(Intern Med 58: 1993-2002, 2019)

(DOI: 10.2169/internalmedicine.2234-18)

\section{Introduction}

Pancreatic ductal adenocarcinoma (PDAC) has the worst survival rate among common cancers; the 3-year survival rate of unresectable PDAC is approximately $3 \%$ (1). The ef- ficacy of FOLFIRINOX (FFX), a regimen consisting of leucovorin (I-LV), 5-fluorouracil (5-FU), irinotecan (CPT-11), and oxaliplatin (L-OHP), was first reported in 2011 for advanced-staged PDAC patients in a phase III trial (ACCORD-11) (2). Although FFX exhibited survival advantages compared with gemcitabine, more adverse events oc-

${ }^{1}$ Department of Gastroenterology and Hepatology, Kindai University Faculty of Medicine, Japan and ${ }^{2}$ Clinical Research Center, Kindai University Hospital, Japan

Received: October 4, 2018; Accepted: January 27, 2019; Advance Publication by J-STAGE: April 17, 2019

Correspondence to Dr. Kentaro Yamao, yamaken_volvo@yahoo.co.jp 
curred in the FFX group than in the gemcitabine group (2). These adverse events included severe neutropenia [grade 3-4 according to the National Cancer Institute Common Terminology Criteria for Adverse Events (CTCAE), version 4. (3)] and febrile neutropenia $(\mathrm{FN})$, both of which reduce the patients' quality of life. Indeed, grade 3-4 neutropenia and FN occurred in $37.3-77.8 \%$ and $5.4-22.2 \%$ of cases, respectively, in previous FFX trials in which patients did not receive prophylactic granulocyte colony-stimulating factor (GCSF) $(2,4,5)$. Such a high incidence of hematotoxicity by FFX prompted oncologists to develop a modified FFX regimen (mFFX) that lacks bolus 5-FU administration and has a decreased CPT-11 dose to reduce neutropenia and FN (6-10). It should be noted, however, that the incidences of grade 3-4 neutropenia and FN were comparable between mFFX and the original full-dose FFX trials in the absence of primary prophylactic G-CSF $(11,12)$. Furthermore, strong myelosuppressive effects, which often accompany the mFFX regimen, require clinicians to reduce the dosage and/ or delay the administration of the next chemotherapy cycle $(4,8,12)$. Maintaining the dose intensity (DI) and chemotherapy schedule is therefore important for maximizing the therapeutic effects.

Pegylated G-CSF (Peg G) is G-CSF that is applicable for prophylaxis against neutropenic complications. Peg $\mathrm{G}$ has a longer half-life than non-pegylated (conventional) G-CSF due to the conjugation of polyethylene glycol (13). Such long-lasting effects of G-CSF on hematopoietic stem cells have been shown to reduce the incidence of neutropenia and FN in patients undergoing moderate- or high-dose myelotoxic chemotherapy (14-24). The American Society of Clinical Oncology (ASCO) (25) and the European Organization for Research and Treatment of Cancer (EORTC) guidelines (26) have recently recommended secondary prophylaxis with G-CSF in patients who exhibit neutropenic events caused by prior cycles of chemotherapy or who require DI maintenance. The purpose of chemotherapy combined with prophylactic G-CSF is to prevent neutropenic events and to ensure that chemotherapy is performed safely and on schedule without dose reductions. In this regard, chemotherapy combined with prophylactic Peg G may prolong the progression-free survival (PFS) and overall survival (OS), as the long-lasting effects of G-CSF by pegylation lead to sustained numbers of functional hematopoietic cells. In fact, the PFS in patients with hematological malignancies was improved by prophylactic Peg G $(27,28)$. However, the clinical safety and efficacy of secondary prophylactic Peg $G$ for mFFX in PDAC remain to be studied.

In this study, we retrospectively investigated the clinical safety and efficacy of mFFX combined with secondary prophylaxis with Peg G in PDAC patients. We explored whether or not combination therapy with mFFX and Peg G prolonged the PFS in PDAC patients who exhibited neutropenic events in previous mFFX cycles.

\section{Materials and Methods}

\section{Patients}

A total of 336 PDAC patients at Kindai University Hospital between January 2014 and July 2018 were identified by medical records. Patients were enrolled if they met all of the following criteria: 1) a pathological PDAC diagnosis; 2) PDAC considered unresectable due to tumor progression; and 3) received more than $2 \mathrm{mFFX}$ cycles and exhibited grade 3-4 neutropenia as defined by $<1,000$ neutrophils per $\mathrm{mm}^{3}$ and/or FN. Patients were excluded if they met any of the following criteria: 1) received full-dose FFX; 2) received 1 or 2 mFFX cycles and had a neutrophil count $<2,000$ per $\mathrm{mm}^{3}$ before $\mathrm{mFFX}$; or 3 ) had uridine diphosphate glucuronosyltransferase (UGT) A1 genetic polymorphisms (homozygous $U G T 1 A 1 * 28$ or $U G T 1 A 1^{*} 6$ or heterozygous $U G T$ $1 A 1 * 6$ or $\left.U G T 1 A 1^{*} 28\right)(29,30)$.

This was a retrospective study, and ethical permission for its performance was obtained from the Review Boards of Kindai University Faculty of Medicine (Registration number: 29-141).

\section{The MFOLFIRINOX regimen}

Patients were treated with mFFX every 2 weeks as follows: L-OHP $\left(85 \mathrm{mg} / \mathrm{m}^{2}\right), I-\mathrm{LV}\left(200 \mathrm{mg} / \mathrm{m}^{2}\right)$ and CPT-11 $\left(150 \mathrm{mg} / \mathrm{m}^{2}\right)$ on day 1 , and then 5 -FU $\left(2,400 \mathrm{mg} / \mathrm{m}^{2}\right)$ on days 1,2 , and 3. Prior to L-OHP administration, the 5hydroxytryptamine (HT) receptor antagonist dexamethasone and a selective neurokinin 1 receptor antagonist were given to all patients for nausea and vomiting. Patients received the mFFX regimen repeatedly, and treatment was repeated unless serious toxicity was observed; discontinuation was decided by the investigators.

\section{Administering Peg $G$ and dose reductions in the mFOLFIRINOX-Peg G group}

Peg G (3.6 mg) administration was initiated 24-72 hours after the 5-FU infusion of the cycle following the observation of a grade 3-4 neutropenic event (neutropenia or FN) in a previous mFFX cycle. Patients who received Peg G were defined as the mFFX-Peg $G$ group (Peg G group). Peg $G$ administration was left to the discretion of each physician and was continued until the final mFFX cycle. Dose reductions of chemotherapeutic agents were performed when grade 3-4 neutropenic events occurred despite Peg G administration or when other adverse events, such as thrombocytopenia, anemia, diarrhea, mucositis, hand and foot syndrome, elevated total bilirubin, or neuropathy, were observed.

Dose reductions were carried out based on the modified criteria of Conroy et al. (2) (Supplementary material). The details of dose reductions and the criteria for reductions are shown in the Supplementary material. In brief, dose reductions of chemotherapeutic agents were performed based on 
hematological and non-hematological toxicities. Toxicities were assessed using the National Cancer Institute CTCAE, version 4 , as described previously (3). In cases of hematological toxicities, dose reductions were performed in accordance with the criteria shown in Supplementary Tables 1 and 2. In cases of non-hematological toxicities, dose reductions were performed in accordance with the criteria shown in Supplementary Table 3. Chemotherapy was delayed until the recovery status met all of the following criteria: neutrophil count $>1,500$ per $\mathrm{mm}^{3}$, platelet count $>75,000$ per $\mathrm{mm}^{3}$, total bilirubin $<1.5 \mathrm{mg} / \mathrm{dL}$, grade $\leq 2$ peripheral sensory neuropathy, and grade $\leq 2$ diarrhea and watery stool.

\section{Dose reductions in the mFOLFIRINOX-non-Peg G group}

In response to various adverse events, including neutropenic events, dose reductions of chemotherapeutic agents were performed without secondary prophylactic Peg G; this group was defined as the mFFX-non-Peg G group (non-Peg $\mathrm{G}$ group). The application criteria of dose reductions and delays were the same as for the Peg G group described above (Supplementary Table 1-3).

\section{The PFS and OS}

To clarify the therapeutic effects of secondary prophylactic Peg $G$ in advanced PDAC patients treated with mFFX, we compared the PFS and OS between the Peg G and nonPeg G groups. The PFS was defined as the time from the initiation of the third cycle to the earliest date of disease progression or death. Cases were censored when tumors were reduced in size enough to allow surgery. The OS was defined as the time from the initiation of the third cycle to death from any cause.

\section{Proportion of adverse events}

The incidence of adverse events, dose reductions, chemotherapy delays, and hospitalizations due to adverse events were compared between the Peg G and non-Peg G groups. The proportion of dose reductions was defined as the proportion of patients who needed dose reductions after the third mFFX cycle. The proportion of chemotherapy delays was defined as the proportion of patients whose chemotherapy could not be started on the prescribed start date after the third cycle. The proportion of hospitalizations was defined as the proportion of patients requiring hospital admission after the third cycle.

\section{Calculating the relative dose intensity (RDI) for each cycle}

The RDI in each cycle was calculated as the ratio of the actual DI in each cycle to the planned DI, as follows: RDI in each cycle $(\%)=$ actual dose in each cycle $\left(\mathrm{mg} / \mathrm{m}^{2}\right) /$ planned dose $\left(\mathrm{mg} / \mathrm{m}^{2}\right) \times 100(\%)$.

\section{Changes in blood cell counts}

Peripheral blood cell counts were compared with the ab- solute blood cell counts on day 1 of each cycle. Numbers of white blood cells (WBCs), neutrophils, monocytes, lymphocytes, basophils, eosinophils, and platelets were determined as previously described (31).

\section{Statistical analyses}

Categorical variables were summarized as the number of events and proportions (\%), and $\mathrm{p}$ values are calculated using Fisher's exact test. Continuous variables were summarized as the means ( \pm standard error), and $\mathrm{p}$ values were calculated using Wilcoxon's rank sum test. Time-to-event variables (OS and PFS) were assessed using the Kaplan-Meier method, and $\mathrm{p}$ values were calculated using the log-rank test. All statistical analyses were performed using GraphPad Prism v.5 software program (GraphPad Software, San Diego, USA).

\section{Results}

\section{Patient characteristics}

The details of patient selection and clinical characteristics of the enrolled cohort are shown in Fig. 1 and Table 1. Grade 3-4 neutropenia or FN occurred in 28 of the 45 PDAC patients who received mFFX. Among these 28 patients, 12 received Peg G (Peg G group), and 16 did not (non-Peg $\mathrm{G}$ group). One in the Peg $\mathrm{G}$ group and three in the non-Peg G group received only one or two mFFX cycles and were excluded from this study. Ultimately, 11 patients in the Peg G group and 13 in the non-Peg G group were included in the final analysis (Fig. 1).

The characteristics of the patients in the Peg $G$ and nonPeg G groups are shown in Table 1. The peripheral blood neutrophil count before mFFX was significantly lower in the Peg $G$ group than in the non-Peg G group, but no significant differences were found between the two groups regarding age, sex, performance status, tumor location, tumor stage, serum levels of carcinoembryonic antigen (CEA) and CA19-9, or UGT1A1 polymorphisms. The median number of mFFX cycles was 8 (range: 4-36) in the Peg G group and 7 (range: $3-17$ ) in the non-Peg G group. In the Peg G group, 11 and 4 patients exhibited grade 3-4 neutropenia and/or FN in the first and second cycles, respectively. Peg G administration was initiated in the third cycle and continued to the end of treatment in all cases in the Peg G group.

\section{The PFS and OS after the third chemotherapy cycle}

To assess the clinical efficacy of combined mFFX and Peg G, we first estimated the PFS and OS after the third chemotherapy cycle in the Peg G and non-Peg G groups. As shown in Fig. 2A, the median PFS after the third chemotherapy cycle in the Peg G-group was 7.0 months, whereas that of the non-Peg $G$ group was 3.1 months. Thus, the combination therapy markedly prolonged the PFS compared with $\mathrm{mFFX}$ alone $(\mathrm{p}=0.02)$. mFFX was continued in four Peg $G$ patients after the observation period, and one Peg G 


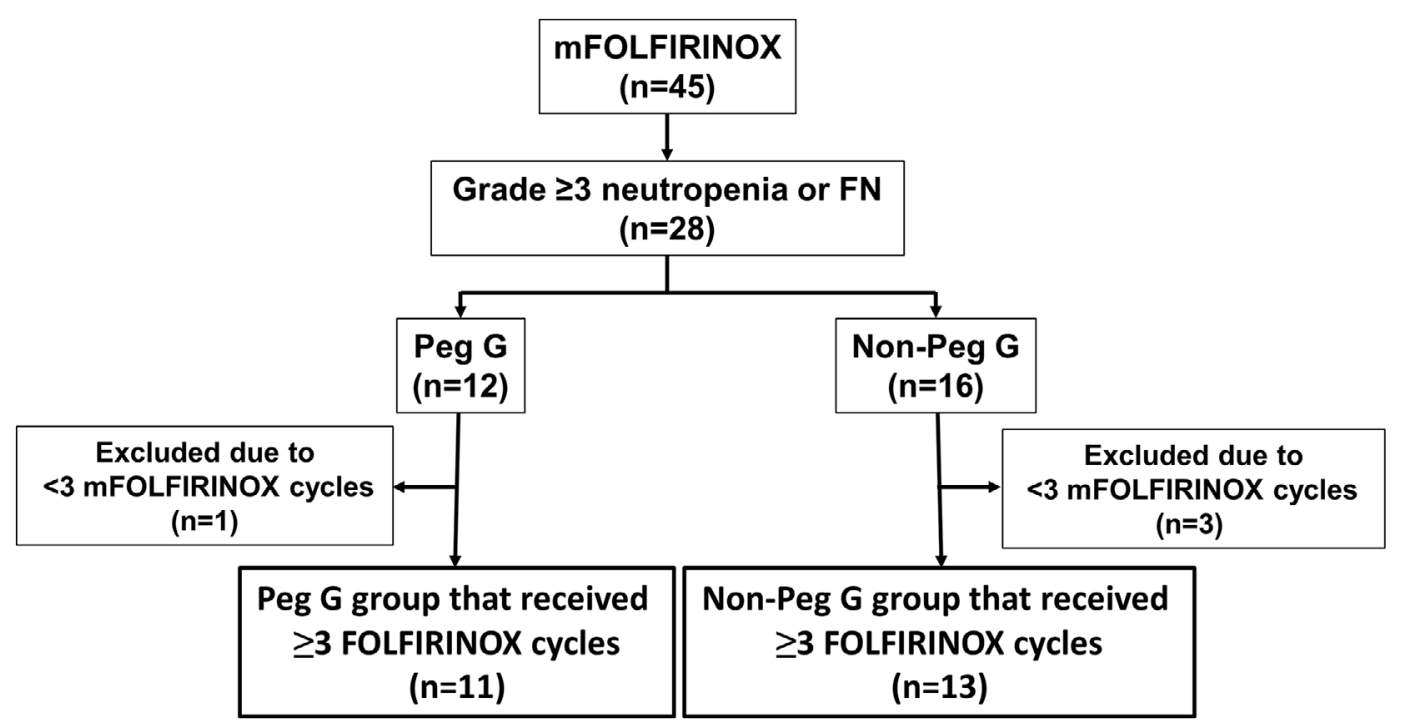

Figure 1. Study flow chart. Forty-five advanced pancreatic ductal adenocarcinoma (PDAC) patients received mFOLFIRINOX (mFFX), with 28 (62.2\%) exhibiting grade 3-4 neutropenia or febrile neutropenia (FN). Twelve of these patients were treated with pegylated G-CSF (Peg G group), and 16 were not (non-Peg G group). One patient in the Peg G group and three in the non-Peg G group received only one or two mFFX cycles and were excluded. Ultimately, 11 patients in the Peg G group and 13 in the non-Peg $G$ group were included in the final analysis.

patient underwent surgical resection after their eighth cycle because of a massive reduction in their primary tumor.

In the Peg $G$ group, the median OS was not reached after the third chemotherapy cycle (survival rates at 6 and 12 months were $81.8 \%$ and $72.7 \%$, respectively), and 7 patients were still alive at the end of the observation period. Conversely, in the non-Peg G group, the median OS after the third chemotherapy cycle was 10.0 months, and only 1 patient was still alive. As shown in Fig. 2B, the OS in the Peg $G$ group was better than that in the non-Peg $G$ group, although this difference was not statistically significant $(\mathrm{p}=$ 0.19).

Taken together, these results strongly suggest that combination therapy with mFFX and Peg G prolonged the PFS in PDAC patients.

\section{Adverse hematological events after the third chemo- therapy cycle}

We next compared incidences of adverse hematological events, as hematotoxicity is a major adverse event of mFFX $(11,12)$. As shown in Table 2,11 cases $(84.6 \%)$ of neutropenic events, including 8 cases of grade 3-4 neutropenia and 3 cases of FN, were seen in the non-Peg G group after the third chemotherapy cycle, while neutropenia was only observed in 1 patient $(9.1 \%)$ with no patients exhibiting grade 3-4 neutropenia or FN in the Peg G group. Thus, the incidence of neutropenic events was much lower in the Peg $G$ group than in the non-Peg $G$ group ( $0 \%$ vs. $61.5 \%$; $\mathrm{p}<0.01)$.

The incidence of grade 3-4 thrombocytopenia was comparable between the Peg G and non-Peg G groups. Severe anemia (grade 3-4) was not observed after the third chemother- apy cycle in either group. Thus, scheduled Peg G administration prevented the development of mFFX-induced neutropenia and FN.

\section{Non-hematological adverse events after the third chemotherapy cycle}

Non-hematological adverse events after the third mFFX cycle are listed in Table 2. Anorexia, fatigue, nausea, diarrhea, and neuropathy were observed in approximately 40$70 \%$ of cases in both groups. There was no significant difference in the incidence of non-hematological adverse events between the two groups. No side effects related to Peg G were observed in the 11 patients in the Peg G group in this study.

Dose reductions, chemotherapy delays, and hospitalizations after the third chemotherapy cycle

The numbers and percentages of patients who required dose reductions, chemotherapy delays, and hospitalizations after the third chemotherapy cycle are shown in Table 3. Four patients $(36.3 \%)$ in the Peg G group required dose reductions: 2 because of thrombocytopenia and 2 because of neuropathy. Chemotherapy was delayed in three of these four patients (for the same reasons). Eight patients (61.5\%) in the non-Peg $\mathrm{G}$ group required dose reductions and chemotherapy delays because of neutropenic events: 6 because of neutropenia and 2 because of FN. Thus, the incidences of dose reductions and chemotherapy delays were lower in the Peg $G$ group than in the non-Peg $G$ group, probably because scheduled Peg G administration effectively prevented neutropenia and FN development. Of note, the incidence of dose reductions and chemotherapy delays due to neutropenic 
Table 1. Patient Characteristics.

\begin{tabular}{|c|c|c|c|}
\hline & Peg G group (n=11) & Non-Peg G group (n=13) & $\mathrm{p}$ value \\
\hline \multicolumn{4}{|l|}{ Sex, n (\%) } \\
\hline Male & $8(72.7)$ & $11(84.6)$ & 0.83 \\
\hline Female & $3(27.2)$ & $2(15.4)$ & \\
\hline \multicolumn{4}{|l|}{ Age, years } \\
\hline Median (range) & $66(53-74)$ & $64(52-70)$ & 0.26 \\
\hline \multicolumn{4}{|c|}{ ECOG performance status, n (\%) } \\
\hline 0 & $7(63.6)$ & $9(69.2)$ & 0.88 \\
\hline 1 & $4(36.3)$ & $4(30.8)$ & \\
\hline \multicolumn{4}{|l|}{ Tumor location, n (\%) } \\
\hline Head & $3(27.2)$ & $4(30.8)$ & 0.94 \\
\hline Body & $3(27.2)$ & $3(23.1)$ & \\
\hline Tail & $2(18.2)$ & $1(7.7)$ & \\
\hline Resected & $3(27.2)$ & $5(38.5)$ & \\
\hline \multicolumn{4}{|l|}{ Body surface area } \\
\hline Median (range) & $1.58(1.21-1.91)$ & $1.73(1.51-2.0)$ & 0.14 \\
\hline \multicolumn{4}{|l|}{ UICC stage, n (\%) } \\
\hline III (Locally advanced) & $3(27.2)$ & $1(7.7)$ & 0.46 \\
\hline IV (Metastatic) & $8(72.7)$ & $12(92.3)$ & \\
\hline \multicolumn{4}{|l|}{ Metastatic sites, n (\%) } \\
\hline No metastasis & $3(27.2)$ & $1(7.7)$ & 0.17 \\
\hline Liver & $6(54.5)$ & $5(38.5)$ & \\
\hline Other site & $2(18.2)$ & $7(53.8)$ & \\
\hline \multicolumn{4}{|l|}{ Baseline neutrophil count } \\
\hline Median (range) & $2,543(2,790-10,400)$ & $3,884(1,577-8,484)$ & 0.31 \\
\hline \multicolumn{4}{|l|}{ CEA at start of FOLFIRINOX } \\
\hline Median (range) & $3.3(1.2-226)$ & $4.2(1.6-37.2)$ & 0.29 \\
\hline \multicolumn{4}{|c|}{ CA19-9 at start of FOLFIRINOX } \\
\hline Median (range) & $63(4-83,895)$ & $458(9-50,081)$ & 0.38 \\
\hline \multicolumn{4}{|l|}{ UGT1A1 $(* 6 / * 28), \mathrm{n}(\%)$} \\
\hline Wild-type/wild-type & $5(45.5)$ & $6(46.2)$ & 1.00 \\
\hline Wild-type/heterozygous & $4(36.3)$ & $5(38.5)$ & \\
\hline Heterozygous/wild-type & $2(18.2)$ & $2(15.4)$ & \\
\hline \multicolumn{4}{|l|}{ mFOLFIRINOX cycles } \\
\hline Median (range) & $9(4-27)$ & $7(3-16)$ & 0.11 \\
\hline \multicolumn{4}{|l|}{ Chemotherapy, n (\%) } \\
\hline First line & $3(27.2)$ & $5(38.5)$ & 0.86 \\
\hline Second line & $6(54.5)$ & $7(53.8)$ & \\
\hline Third line & $2(18.1)$ & $1(7.7)$ & \\
\hline
\end{tabular}

events was significantly different between the two groups.

One patient $(9.1 \%)$ in the Peg G group required hospitalization because of hyperglycemia, whereas $5(38.5 \%)$ in the non-Peg $\mathrm{G}$ group required hospitalization. The reasons for hospitalization in the non-Peg $\mathrm{G}$ group included FN (two cases), cholangitis without a neutropenic event (one case), small bowel obstruction due to tumor invasion (one case), and cerebral infarction (one case). Collectively, these data suggest that combination therapy of mFFX and Peg G reduced the incidences of dose reductions, chemotherapy delays, and hospitalizations by preventing neutropenic events.

\section{RDI of chemotherapeutic agents}

We next compared the RDI in each cycle between the Peg $\mathrm{G}$ and non-Peg $\mathrm{G}$ groups. As shown in Fig. 3, no significant difference was seen in the RDI of L-OHP, $I-\mathrm{LV}$, or 5-FU be- tween these two groups throughout the observation period. In contrast, the RDI of CPT-11 was significantly higher in the Peg G group than in the non-Peg G group at the 6th-9th chemotherapy cycles. These RDI data suggest that the prolonged PFS of PDAC patients treated with both mFFX and Peg G was associated with a higher CPT-11 RDI.

\section{Altered blood cell counts after chemotherapy}

As scheduled Peg G administration markedly reduced neutropenic events and maintained the CPT-11 RDI, we next determined the effects of Peg $G$ on the numbers of peripheral blood hematopoietic cells. As shown in Fig. 4A and B, the numbers of WBCs and neutrophils were significantly higher in the Peg G group than in the non-Peg G group throughout the observation period. Interestingly, the numbers of monocytes were also significantly higher in the Peg G 
A

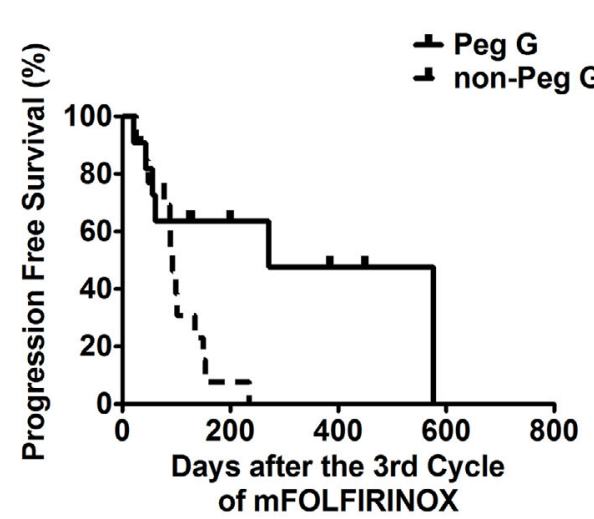

B

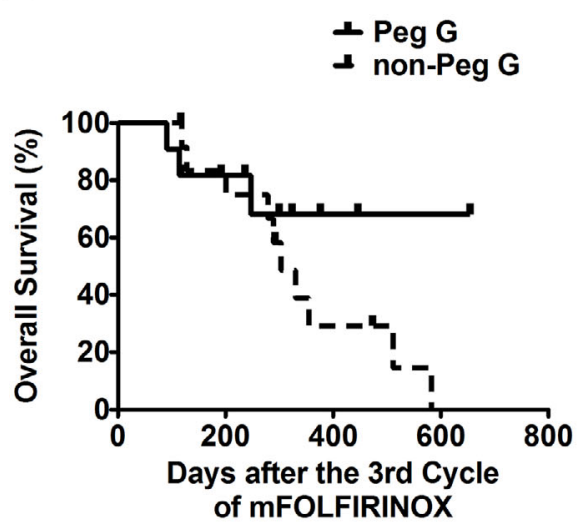

Figure 2. Kaplan-Meier curves of the progression-free survival and overall survival after the third chemotherapy cycle. The progression-free survival (PFS, A) and overall survival (OS, B) in pancreatic ductal adenocarcinoma patients treated with (Peg G group) or without (non-Peg G group) pegylated G-CSF (Peg G). The PFS in the Peg G group was significantly longer than in the non-Peg G group; *p $<\mathbf{0 . 0 5}$.

Table 2. Hematological and Non-hematological Adverse Events after the Third Chemotherapy Cycle.

\begin{tabular}{lccccc}
\hline & \multicolumn{2}{c}{ Peg G group $(\mathrm{n}=11)$} & \multicolumn{2}{c}{ Non-Peg G group $(\mathrm{n}=13)$} & p value \\
\cline { 2 - 6 } & All grades & Grade 3-4 & All grades & Grade 3-4 & Grade 3-4 \\
\hline Hematological event & & & & & \\
Neutropenia & $1(9.1)$ & $0(0)$ & $11(84.6)$ & $8(61.5)$ & $<0.01$ \\
Febrile neutropenia & $0(0)$ & $0(0)$ & $3(23.1)$ & $3(23.1)$ & 0.28 \\
Thrombocytopenia & $4(36.3)$ & $2(18.2)$ & $5(38.5)$ & $0(0)$ & 0.39 \\
Anemia & $2(18.2)$ & $0(0)$ & $1(7.7)$ & $0(0)$ & NA \\
\hline Non-hematological event & & & & & \\
Anorexia & $7(63.4)$ & $0(0)$ & $10(76.9)$ & $0(0)$ & NA \\
Fatigue & $8(72.7)$ & $1(9.1)$ & $7(53.8)$ & $2(15.4)$ & 0.93 \\
Nausea & $4(36.3)$ & $1(9.1)$ & $6(46.2)$ & $1(9.1)$ & 0.54 \\
Vomiting & $0(0)$ & $0(0)$ & $0(0)$ & $0(0)$ & NA \\
Diarrhea & $6(54.5)$ & $2(18.2)$ & $6(46.2)$ & $3(23.1)$ & 0.83 \\
Mucositis & $1(9.1)$ & $0(0)$ & $3(23.1)$ & $0(0)$ & NA \\
Neuropathy & $5(54.5)$ & $2(18.2)$ & $6(46.2)$ & $2(15.4)$ & 0.71 \\
Hyperglycemia & $1(9.1)$ & $1(9.1)$ & $0(0)$ & $0(0)$ & 0.89 \\
\hline
\end{tabular}

Table 3. Dose Reductions, Chemotherapy Delays and Hospitalizations after the Third Chemotherapy Cycle.

\begin{tabular}{|c|c|c|c|}
\hline & Peg G group $(n=11)$ & Non-Peg G group $(n=13)$ & $\mathrm{p}$ value \\
\hline Dose reductions for any reason & $4(36.3)$ & $8(61.5)$ & 0.41 \\
\hline Dose reductions because of neutropenic events* & $0(0)$ & $8(61.5)$ & $<0.01$ \\
\hline Dose delays for any reason & $3(27.2)$ & $8(61.5)$ & 0.20 \\
\hline Dose delays because of neutropenic events* & $0(0)$ & $8(61.5)$ & $<0.01$ \\
\hline Hospitalizations for any reason & $1(9.1)$ & $5(38.5)$ & 0.24 \\
\hline Hospitalizations for neutropenic events* & $0(0)$ & $2(15.4)$ & 0.54 \\
\hline
\end{tabular}

*Neutropenia and febrile neutropenia

group than in the non-Peg $\mathrm{G}$ group throughout the observation period (Fig. 4C). In contrast, no significant differences were seen in the numbers of lymphocytes, basophils, eosinophils, or platelets between the two groups (Fig. 4D-G).
Thus, scheduled Peg G administration sustained the numbers of peripheral blood neutrophils and monocytes but not lymphocytes. Together, these data suggest that the sustained numbers of peripheral blood neutrophils and monocytes in- 

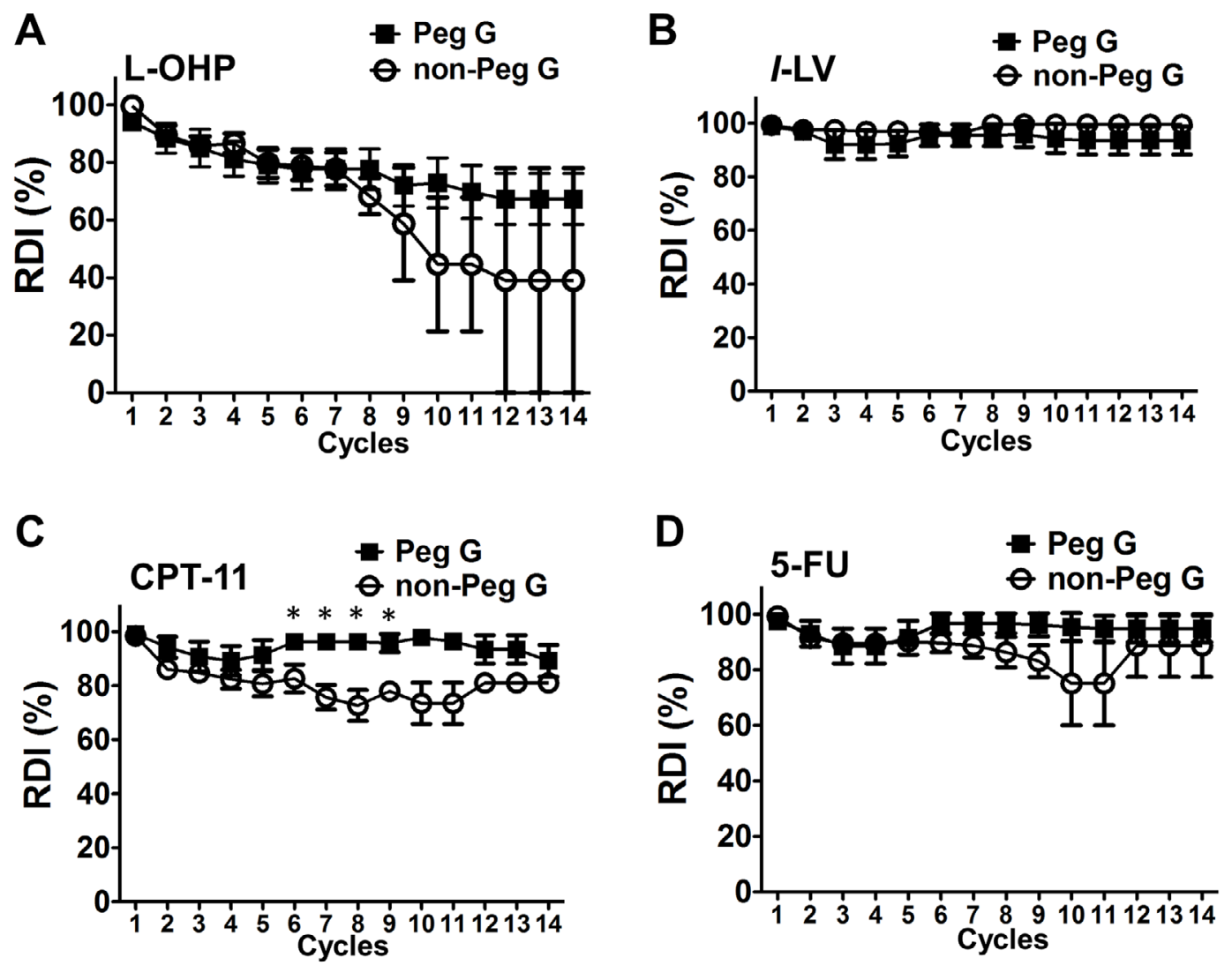

Figure 3. Changes in the relative dose intensity at each mFOLFIRINOX cycle. The relative dose intensities (RDIs) of the four chemotherapeutic agents of mFFX were determined at each chemotherapy cycle. The RDIs for leucovorin (I-LV), 5-fluorouracil (5-FU), irinotecan (CPT-11), and oxaliplatin (L-OHP) are shown. Results are shown as the mean \pm standard error; * $\mathbf{p}<0.05$.

duced by Peg $G$ are associated with a prolonged PFS in PDAC patients treated with $\mathrm{mFFX}$ due to maintenance of the high RDI of CPT- 11 .

\section{Discussion}

In this study, we showed that scheduled secondary prophylactic Peg $G$ administration prolonged the PFS in advanced PDAC patients treated with mFFX. The prolonged PFS due to scheduled Peg G administration was accompanied by sustained numbers of peripheral blood neutrophils and monocytes, which might prevent neutropenic events as well as allow for a high RDI of CPT-11, a key component of the mFFX regimen.

Short-half-life G-CSF (non-pegylated) is widely used as a supportive therapy for recovery from neutropenia and FN in cancer patients receiving moderate- or high-dose myelotoxic agents $(15,32)$. The clinical guidelines from the ASCO and EORTC recommend primary prophylaxis with G-CSF when a patient's overall FN risk is greater than 20\%. In addition to primary prophylaxis, secondary prophylaxis with G-CSF is recommended when patients experience neutropenic events from prior chemotherapy cycles and require highlevel RDIs to obtain maximal therapeutic effects from chemotherapy $(25,26)$. It is generally established that maximal therapeutic effects of chemotherapy can be obtained by pre- venting adverse effects, such as neutropenia and FN, as well as by maintaining high RDIs. The scheduled secondary administration of prophylactic Peg G may be attractive for preventing neutropenic events, as this treatment leads to sustained numbers of peripheral blood neutrophils through the long-lasting effects of G-CSF (13). Indeed, scheduled Peg G administration prolonged the PFS in patients with hematological malignancies $(27,28)$. Although the mFFX regimen is widely used because of its efficacy in PDAC patients, dose reductions and/or chemotherapy delays are often required due to the high incidence of adverse events, especially neutropenia and FN. In this study, we tried to retrospectively determine the clinical safety and efficacy of scheduled secondary prophylactic Peg $G$ administration in PDAC patients who received mFFX. Surprisingly, we found that scheduled Peg G administration markedly reduced the incidences of neutropenic events associated with mFFX due to the sustained numbers of peripheral blood neutrophils and ultimately prolonged the PFS (Table 2 and Fig. 2A). Previous reports have shown that repeated Peg G administration caused severe adverse events specific to Peg G, such as spontaneous splenic rupture (33), musculoskeletal pain (34-38), and interstitial pneumonia. These severe adverse effects were not observed in the Peg G group in this study.

To our knowledge, this is the first report to show the 


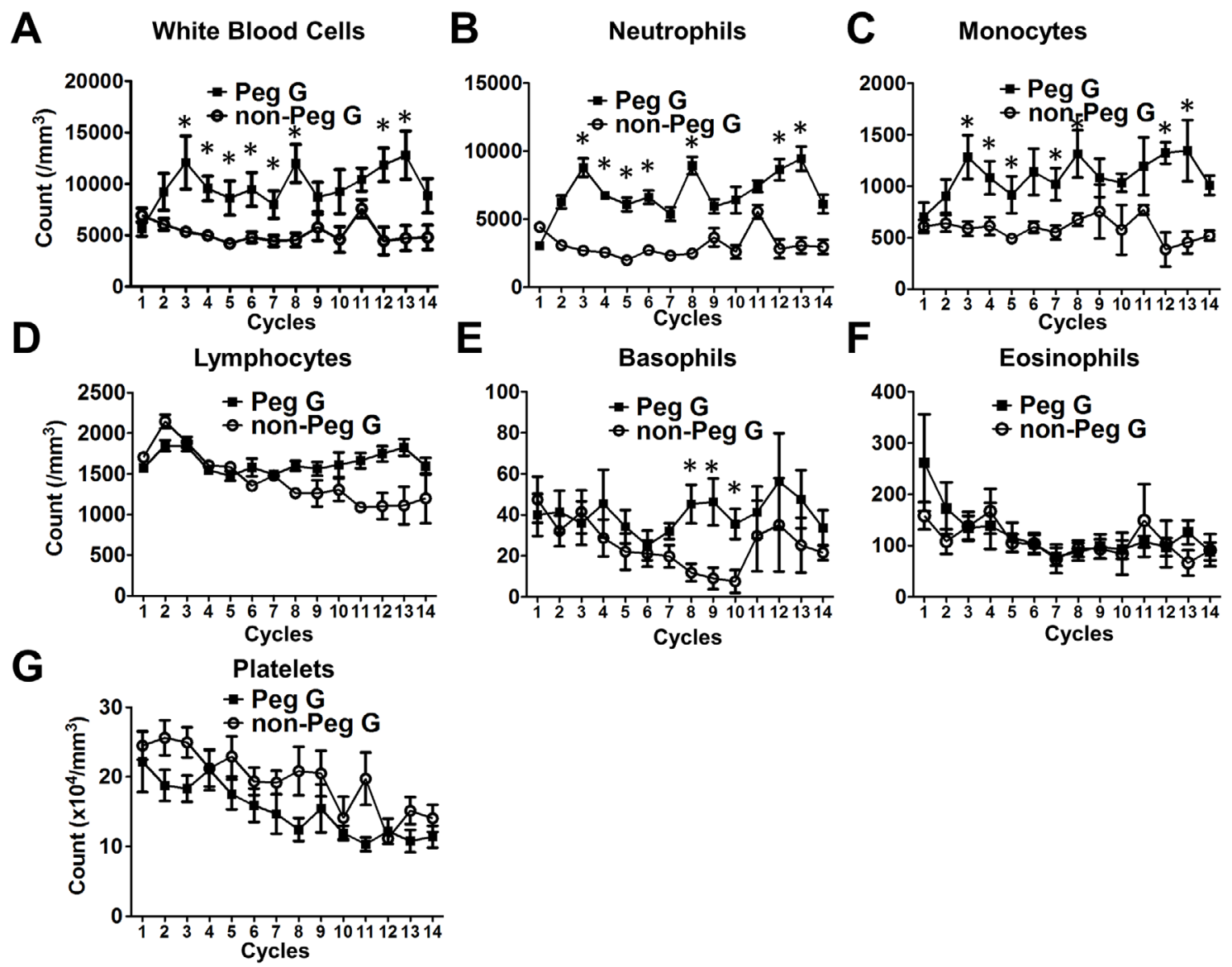

Figure 4. Changes in peripheral blood hematopoietic cells at each mFOLFIRINOX cycle. Numbers of peripheral blood white blood cells (WBCs), neutrophils, monocytes, lymphocytes, basophils, eosinophils, and platelets are shown. Results are shown as the mean \pm standard error; $* \mathbf{p}<0.05$.

clinical advantages of scheduled Peg $G$ administration in mFFX-treated PDAC patients. The OS was also longer in the Peg G group than in the non-Peg G group, although this was not statistically significant. However, it should be noted that a significant number of patients in the Peg G group continued mFFX after the observation period, whereas most of the patients in the non-Peg G group died. Therefore, it may be too early to determine the OS in this study. Whether or not scheduled Peg $\mathrm{G}$ administration prolongs not only the PFS but also the OS will be explored in our ongoing, largescale prospective study.

Achieving the planned RDI is important for obtaining the maximum therapeutic effects of chemotherapy (39). In fact, a high chemotherapy RDI has been shown to be associated with improved clinical outcomes in a few previous reports $(40,41)$. Consistent with this idea, the RDI of a key mFFX component, CPT-11, showed higher trends in the Peg $\mathrm{G}$ group than in the non-Peg G group (Fig. 3). In contrast, the RDIs of the other three components-L-OHP, I-LV, and 5FU-were comparable between the two groups. The higher CPT-11 RDI may be explained by the fact that dose reductions of CPT-11 are required upon encountering neutropenia and FN (Supplementary Table 2). Therefore, it is likely that preventing neutropenia and FN through scheduled Peg G administration resulted in a prolonged PFS for PDAC patients partially through the achievement of a higher CPT-11 RDI.

As expected, scheduled Peg G administration induced an expansion of peripheral blood neutrophils throughout the observation period. Our extensive analysis regarding changes in the peripheral blood immune cell subpopulations revealed that scheduled Peg $G$ administration led to expansions of neutrophils and monocytes but not lymphocytes, basophils, or eosinophils (Fig. 4). Thus, sustained numbers of peripheral blood neutrophils and monocytes are a characteristic alteration of peripheral blood immune cells associated with scheduled Peg G administration. It is without a doubt that the Peg G-mediated expansion of neutrophils contributed to the clinical advantage and maintenance of high RDIs in this study. However, whether or not the prolonged PFS observed in this study can be solely attributed to the ability to maintain high-level RDIs is unclear.

In this regard, the Peg G-induced expansion of peripheral blood neutrophils and monocytes prompted us to speculate that long-lasting G-CSF might promote the differentiation of myeloid cells with potent antitumor immunity. Tumorinfiltrating myeloid-derived suppressor cells (MDSCs) and tumor-associated macrophages (TAMs), both of which are characterized by the capacity to suppress $\mathrm{T}$ and natural killer (NK) cell cancer immune surveillance, promote cancer progression $(42,43)$. In fact, MDSCs and TAMs are key play- 
ers in the pancreatic cancer microenvironment that support tumor progression and dissemination $(44,45)$. Monocytes are immature myeloid cells that can differentiate into macrophages and dendritic cells $(46,47)$. Cytokines and chemokines released into the cancer microenvironment regulate the differentiation of monocytes into M1 macrophages and M2 macrophages, with tumor-suppressive and tumor-promoting activities, respectively (42-47). Given the fact that scheduled Peg $G$ administration led to an expansion of not only neutrophils but also monocytes, it would be interesting to see which types of macrophages (M1 vs. M2) accumulated in the tumors of PDAC patients treated with mFFX and Peg G.

Two concerns need to be addressed before the scheduled administration of secondary prophylactic Peg $\mathrm{G}$ is applied to PDAC patients treated with mFFX. First, the cost of repeated Peg $\mathrm{G}$ administration cannot be ignored, as it is an expensive agent. For this reason, Peg G application as primary prophylaxis may not be feasible for all patients receiving mFFX. Second, patients are usually required to revisit the hospital for Peg $G$ administration. To overcome this issue, Peg G has been administered on the same day as chemotherapy in some clinical trials, which resulted in a higher incidence of $\mathrm{FN}$ than with administration a few days after completing chemotherapy $(48,49)$.

Despite the careful and intense analysis performed in this study, some limitations bear mentioning, and we need to be cautious regarding the interpretation of the results in this study. First, this was a non-randomized, retrospective study. Second, the sample size was relatively small. Therefore, a prospective study with a larger number of patients is required to verify that the scheduled administration of secondary prophylactic Peg G prolongs the PFS in mFFX-treated PDAC patients. Third, the administration of Peg G depended on each physician's decision in this study. There might therefore have been some degree of selection bias for the patients. Whether or not mFFX combined with Peg G prolongs the PFS and/or OS in patients with advanced PDAC should be explored in prospective studies enrolling a large number of patients.

In conclusion, the scheduled administration of secondary prophylactic Peg G prolonged the PFS in PDAC patients treated with the mFFX regimen by preventing dose reductions and chemotherapy delays. The RDI of a key mFFX component, CPT-11, was maintained at high levels due to the sustained numbers of neutrophils in PDAC patients who received scheduled Peg G administration. The scheduled administration of secondary prophylactic Peg $G$ may be recommended for PDAC patients who exhibit grade 3-4 neutropenic events after previous mFFX cycles.

The authors state that they have no Conflict of Interest (COI).

\section{Acknowledgement}

We thank Hitoshi Imaoka for editing a draft of this manuscript.

\section{References}

1. Egawa S, Toma H, Ohigashi $\mathrm{H}$, et al. Japan Pancreatic Cancer Registry; 30th year anniversary: Japan Pancreas Society. Pancreas 41: 985-992, 2012.

2. Conroy T, Desseigne F, Ychou M, et al. FOLFIRINOX versus gemcitabine for metastatic pancreatic cancer. N Engl J Med 364: 1817-1825, 2011.

3. National Cancer Institute Common Terminology Criteria for Adverse Events v. https://evs.nci.nih.gov/ftp1/CTCAE/CTCAE_4.03/C TCAE_4.03_2010-06-14_QuickReference_5x7.pdf

4. Okusaka T, Ikeda M, Fukutomi A, et al. Phase II study of FOLFIRINOX for chemotherapy-naive Japanese patients with metastatic pancreatic cancer. Cancer Sci 105: 1321-1326, 2014.

5. Chllamma MK, Cook N, Dhani NC, et al. FOLFIRINOX for advanced pancreatic cancer: the Princess Margaret Cancer Centre experience. Br J Cancer 115: 649-654, 2016.

6. Bai X, Su R, Ma T, et al. Modified FOLFIRINOX for advanced pancreatic cancer: a tertiary center experience from China. Zhonghua Wai Ke Za Zhi [Chinese Journal of Surgery] 54: 270275, 2016 (in Chinese, Abstract in English).

7. Blazer M, Wu C, Goldberg RM, et al. Neoadjuvant modified (m) FOLFIRINOX for locally advanced unresectable (LAPC) and borderline resectable (BRPC) adenocarcinoma of the pancreas. Ann Surg Oncol 22: 1153-1159, 2015.

8. Ghorani E, Wong HH, Hewitt C, Calder J, Corrie P, Basu B. Safety and efficacy of modified FOLFIRINOX for advanced pancreatic adenocarcinoma: a UK single-centre experience. Oncology 89: 281-287, 2015.

9. Stein SM, James ES, Deng Y, et al. Final analysis of a phase II study of modified FOLFIRINOX in locally advanced and metastatic pancreatic cancer. Br J Cancer 114: 737-743, 2016.

10. Umemura A, Nitta H, Sasaki A, Takahara T, Hasegawa $Y$, Wakabayashi G. Modified FOLFIRINOX for locally advanced and metastatic pancreatic cancer patients resistant to gemcitabine and S-1 in Japan: a single institutional experience. Hepatogastroenterology 61: 814-820, 2014.

11. Li X, Ma T, Zhang Q, et al. Modified-FOLFIRINOX in metastatic pancreatic cancer: a prospective study in Chinese population. Cancer Lett 406: 22-26, 2017.

12. Yoshida $\mathrm{K}$, Iwashita $\mathrm{T}$, Uemura $\mathrm{S}$, et al. A multicenter prospective phase II study of first-line modified FOLFIRINOX for unresectable advanced pancreatic cancer. Oncotarget 8: 111346111355, 2017.

13. Yang BB, Kido A. Pharmacokinetics and pharmacodynamics of pegfilgrastim. Clin Pharmacokinet 50: 295-306, 2011.

14. Vose JM, Crump M, Lazarus H, et al. Randomized, multicenter, open-label study of pegfilgrastim compared with daily filgrastim after chemotherapy for lymphoma. J Clin Oncol 21: 514-519, 2003.

15. Vogel CL, Wojtukiewicz MZ, Carroll RR, et al. First and subsequent cycle use of pegfilgrastim prevents febrile neutropenia in patients with breast cancer: a multicenter, double-blind, placebocontrolled phase III study. J Clin Oncol 23: 1178-1184, 2005.

16. Pinter T, Klippel Z, Cesas A, et al. A phase III, randomized, double-blind, placebo-controlled trial of pegfilgrastim in patients receiving first-line FOLFOX/bevacizumab or FOLFIRI/bevacizumab for locally advanced or metastatic colorectal cancer: final results of the pegfilgrastim and anti-VEGF evaluation study (PAVES). Clin Colorectal Cancer 16: 103-114.e103, 2017.

17. Naeim A, Henk HJ, Becker L, et al. Pegfilgrastim prophylaxis is associated with a lower risk of hospitalization of cancer patients than filgrastim prophylaxis: a retrospective United States claims analysis of granulocyte colony-stimulating factors (G-CSF). BMC Cancer 13: 11, 2013.

18. Kosaka Y, Rai Y, Masuda N, et al. Phase III placebo-controlled, 
double-blind, randomized trial of pegfilgrastim to reduce the risk of febrile neutropenia in breast cancer patients receiving docetaxel/ cyclophosphamide chemotherapy. Support Care Cancer 23: 1137$1143,2015$.

19. Holmes FA, O'Shaughnessy JA, Vukelja S, et al. Blinded, randomized, multicenter study to evaluate single administration pegfilgrastim once per cycle versus daily filgrastim as an adjunct to chemotherapy in patients with high-risk stage II or stage III/IV breast cancer. J Clin Oncol 20: 727-731, 2002.

20. Holmes FA, Jones SE, O'Shaughnessy J, et al. Comparable efficacy and safety profiles of once-per-cycle pegfilgrastim and daily injection filgrastim in chemotherapy-induced neutropenia: a multicenter dose-finding study in women with breast cancer. Ann Oncol 13: 903-909, 2002.

21. Grigg A, Solal-Celigny P, Hoskin P, et al. Open-label, randomized study of pegfilgrastim vs. daily filgrastim as an adjunct to chemotherapy in elderly patients with non-Hodgkin's lymphoma. Leuk Lymphoma 44: 1503-1508, 2003.

22. Green MD, Koelbl H, Baselga J, et al. A randomized double-blind multicenter phase III study of fixed-dose single-administration pegfilgrastim versus daily filgrastim in patients receiving myelosuppressive chemotherapy. Ann Oncol 14: 29-35, 2003.

23. Bondarenko I, Gladkov OA, Elsaesser R, Buchner A, Bias P. Efficacy and safety of lipegfilgrastim versus pegfilgrastim: a randomized, multicenter, active-control phase 3 trial in patients with breast cancer receiving doxorubicin/docetaxel chemotherapy. BMC Cancer 13: 386, 2013.

24. Balducci L, Al-Halawani $\mathrm{H}$, Charu V, et al. Elderly cancer patients receiving chemotherapy benefit from first-cycle pegfilgrastim. Oncologist 12: 1416-1424, 2007.

25. Smith TJ, Bohlke K, Lyman GH, et al. Recommendations for the use of WBC growth factors: American Society of Clinical Oncology Clinical Practice Guideline Update. J Clin Oncol 33: 31993212, 2015.

26. Aapro MS, Bohlius J, Cameron DA, et al. 2010 update of EORTC guidelines for the use of granulocyte-colony stimulating factor to reduce the incidence of chemotherapy-induced febrile neutropenia in adult patients with lymphoproliferative disorders and solid tumours. Eur J Cancer 47: 8-32, 2011.

27. Pfreundschuh M, Trumper L, Kloess M, et al. Two-weekly or 3weekly CHOP chemotherapy with or without etoposide for the treatment of elderly patients with aggressive lymphomas: results of the NHL-B2 trial of the DSHNHL. Blood 104: 634-641, 2004.

28. Gruber M, Fleiss K, Porpaczy E, et al. Prolonged progression-free survival in patients with chronic lymphocytic leukemia receiving granulocyte colony-stimulating factor during treatment with fludarabine, cyclophosphamide, and rituximab. Ann Hematol 90: 11311136, 2011.

29. Yamamoto N, Takahashi T, Kunikane H, et al. Phase I/II pharmacokinetic and pharmacogenomic study of UGT1A1 polymorphism in elderly patients with advanced non-small cell lung cancer treated with irinotecan. Clin Pharmacol Ther 85: 149-154, 2009.

30. Minami H, Sai K, Saeki M, et al. Irinotecan pharmacokinetics/ pharmacodynamics and UGT1A genetic polymorphisms in Japanese: roles of UGT1A1*6 and "28. Pharmacogenet Genomics 17: 497-504, 2007.

31. Chung H, Watanabe T, Kudo M, Chiba T. Correlation between hyporesponsiveness to Toll-like receptor ligands and liver dysfunction in patients with chronic hepatitis $\mathrm{C}$ virus infection. J Viral Hepat 18: e561-e567, 2011.

32. Timmer-Bonte JN, Adang EM, Smit HJ, et al. Cost-effectiveness of adding granulocyte colony-stimulating factor to primary prophylaxis with antibiotics in small-cell lung cancer. J Clin Oncol
24: 2991-2997, 2006.

33. Arshad M, Seiter K, Bilaniuk J, et al. Side effects related to cancer treatment: case 2. Splenic rupture following pegfilgrastim. J Clin Oncol 23: 8533-8534, 2005.

34. Gavioli E, Abrams M. Prevention of granulocyte-colony stimulating factor (G-CSF) induced bone pain using double histamine blockade. Support Care Cancer 25: 817-822, 2017.

35. Moukharskaya J, Abrams DM, Ashikaga T, et al. Randomized phase II study of loratadine for the prevention of bone pain caused by pegfilgrastim. Support Care Cancer 24: 3085-3093, 2016.

36. Bondarenko IM, Bias P, Buchner A. Incidence of bone pain in patients with breast cancer treated with lipegfilgrastim or pegfilgrastim: an integrated analysis from phase II and III studies. Support Care Cancer 24: 267-273, 2016.

37. Kubista E, Glaspy J, Holmes FA, Green MD, Hackett J, Neumann T. Bone pain associated with once-per-cycle pegfilgrastim is similar to daily filgrastim in patients with breast cancer. Clin Breast Cancer 3: 391-398, 2003.

38. Moore DC, Pellegrino AE. Pegfilgrastim-induced bone pain: a review on incidence, risk factors, and evidence-based management. Ann Pharmacother 51: 797-803, 2017.

39. Lyman GH. Impact of chemotherapy dose intensity on cancer patient outcomes. J Natl Compr Canc Netw 7: 99-108, 2009.

40. Chirivella I, Bermejo B, Insa A, et al. Optimal delivery of anthracycline-based chemotherapy in the adjuvant setting improves outcome of breast cancer patients. Breast Cancer Res Treat 114: 479-484, 2009.

41. Budman DR, Berry DA, Cirrincione CT, et al. Dose and dose intensity as determinants of outcome in the adjuvant treatment of breast cancer. The Cancer and Leukemia group B. J Nati Cancer Inst 90: 1205-1211, 1998.

42. Zhao Y, Wu T, Shao S, Shi B, Zhao Y. Phenotype, development, and biological function of myeloid-derived suppressor cells. Oncoimmunology 5: e1004983, 2016.

43. Gabrilovich DI, Nagaraj S. Myeloid-derived suppressor cells as regulators of the immune system. Nat Rev Immunol 9: 162-174, 2009.

44. Stromnes IM, Brockenbrough JS, Izeradjene K, et al. Targeted depletion of an MDSC subset unmasks pancreatic ductal adenocarcinoma to adaptive immunity. Gut 63: 1769-1781, 2014.

45. Zhu Y, Herndon JM, Sojka DK, et al. Tissue-resident macrophages in pancreatic ductal adenocarcinoma originate from embryonic hematopoiesis and promote tumor progression. Immunity 47: 323338.e326, 2017.

46. Solinas G, Germano G, Mantovani A, Allavena P. Tumorassociated macrophages (TAM) as major players of the cancerrelated inflammation. J Leukoc Biol 86: 1065-1073, 2009.

47. Takeya M, Komohara Y. Role of tumor-associated macrophages in human malignancies: friend or foe? Pathol Int 66: 491-505, 2016.

48. Hayama T, Sakurai K, Miura K, et al. Optimal timing for pegfilgrastim administration in Japanese breast cancer patients receiving intermediate-risk chemotherapies. Int J Clin Pharm 40: 997-1000, 2018.

49. Weycker D, Li X, Figueredo J, Barron R, Tzivelekis S, Hagiwara M. Risk of chemotherapy-induced febrile neutropenia in cancer patients receiving pegfilgrastim prophylaxis: does timing of administration matter? Support Care Cancer 24: 2309-2316, 2016.

The Internal Medicine is an Open Access journal distributed under the Creative Commons Attribution-NonCommercial-NoDerivatives 4.0 International License. To view the details of this license, please visit (https://creativecommons.org/licenses/ by-nc-nd/4.0/).

(C) 2019 The Japanese Society of Internal Medicine Intern Med 58: 1993-2002, 2019 